\title{
Functional Equations and Fusion Matrices for the Eight Vertex Model $^{\dagger}$
}

\author{
By \\ Klaus FABRICIUs* and Barry M. MCCoY**
}

\begin{abstract}
We derive sets of functional equations for the eight vertex model by exploiting an analogy with the functional equations of the chiral Potts model. From these equations we show that the fusion matrices have special reductions at certain roots of unity. We explicitly exhibit these reductions for the 3,4 and 5 order fusion matrices and compare our formulation with the algebra of Sklyanin.
\end{abstract}

\section{$\S 1$. Introduction}

The solution of the 8 vertex model by Baxter [1] has many inventive steps. First of all the eigenvalues of the transfer matrix $T$ are studied without having any information on the eigenvectors. Secondly these eigenvalues are computed by inventing a new auxiliary matrix (called $Q$ ) which commutes with $T$ and which satisfies a functional equation with $T$. This functional equation has the further property that it can be used to find all eigenvalues of $T$. The determination of these eigenvalues is often what is called "solving the 8 vertex model".

Many years after the eigenvalues of the 8 vertex model transfer matrix were computed a more elaborate model called the chiral Potts model was discovered to be integrable [2]. For this model there is also an auxiliary matrix $\mathrm{Q}$

Communicated by T. Kawai. Received November 4, 2003.

2000 Mathematics Subject Classification(s): 82B23

${ }^{\dagger}$ This article is an invited contribution to a special issue of Publications of RIMS commemorating the fortieth anniversary of the founding of the Research Institute for Mathematical Sciences.

*Physics Department, University of Wuppertal, 42097 Wuppertal, Germany e-mail: Fabricius@theorie.physik.uni-wuppertal.de

** Institute for Theoretical Physics, State University of New York, Stony Brook, NY 11794-3840

e-mail: mccoy@insti.physics.sunysb.edu

(C) 2004 Research Institute for Mathematical Sciences, Kyoto University. All rights reserved. 
which satisfies a functional equation [3] with the transfer matrix but unlike the 8 vertex model this functional equation is not sufficient to compute the eigenvalues of the transfer matrix. However, further functional equations were first conjectured $[4,5]$ and then proven true [6] from which the desired eigenvalues are computed.

There are in fact many matrices $Q$ which satisfy the $T Q$ equations first discovered [1] in 1972 for the special "root of unity" case

$$
\eta=\frac{m_{1} K}{L}
$$

where the parameter $\eta$ of the 8 vertex model is a rational multiple of $K$ the real period of the elliptic functions. In 1973 Baxter [7-9] found a second matrix $Q$ which satisfies the $T Q$ equations in the root of unity case and also for the case of generic $\eta$. We will here concentrate on the $Q$ matrix of the 1972 paper [1] which we denote by $Q_{72}$.

Recently we have discovered [10] that in the root of unity case (1.1) the functional equations discovered by Baxter [1] do not in fact exhaust the totality of functional equations of the 8 vertex model and we conjectured a functional equation [10] obeyed by $Q_{72}$ alone which allows the computation of the eigenvalues of $Q_{72}$ without reference to $T$. When (1.1) holds many of the eigenvalues of $T$ are degenerate and Baxter's $T Q$ functional equation is not sufficient to compute the degeneracy of the eigenvalues. However the auxiliary matrix $Q_{72}$ is non degenerate and the new functional equation of ref. [10] allows the complete determination of the spectrum of $T$ including the degeneracies.

In the course of searching for a proof of the conjectured functional equation of ref. [10] it has become clear that the 8 vertex model has several more functional equations than were originally found in ref. [1] and in fact there is a complete analogy between the 8 vertex model and all the functional equations found in ref. [6] for the chiral Potts model. The purpose of this paper is to present this analogy.

The concept of the fusion hierarchy [11-17] will play an important role in the analogy and will allow us to find a new functional equation for the 8 vertex transfer matrix which has not yet appeared in the literature. Curiously enough, for the chiral Potts model the fusion matrices have been explicitly computed in ref. [6] for all levels of fusion while for the 8 vertex model we are at present only able to compute explicit formulas for the fusion levels 3,4 and 5 .

In Sec. 2 we review the derivation of the $T Q$ functional equation of Baxter. In Sec. 3 we present the analogy with the chiral Potts model in detail and derive functional equations for the 8 vertex model. In Sec. 4 we explicitly 
compute the fusion matrices for levels 3,4 and 5 . In Sec. 5 we introduce a similarity transformation which makes explicit the degeneration which takes place in $T^{(L+1)}(v)$ when $\eta$ satisfies the root of unity condition (1.1). In Sec. 6 we compare our results with the algebra of Sklyanin [13,14]. We close in Sec. 7 with a proof of the functional equation of ref. [10] for the case of $L=2$.

\section{$\S 2 . \quad$ Baxter's $T Q$ Equation}

We begin our considerations by reviewing Baxter's derivation of the $T Q$ functional equation of ref. [1]. The transfer matrix for the eight vertex model with $N$ columns and periodic boundary conditions is

$$
\left.T_{8}(u)\right|_{\mu, \nu}=\operatorname{Tr} W_{8}\left(\mu_{1}, \nu_{1}\right) W_{8}\left(\mu_{2}, \nu_{2}\right) \cdots W_{8}\left(\mu_{N}, \nu_{N}\right)
$$

where in the conventions of (6.2) of ref. [1].

$$
\begin{aligned}
& \left.W_{8}(1,1)\right|_{1,1}=\left.W_{8}(-1,-1)\right|_{-1,-1}=\Theta(2 \eta) \Theta(v-\eta) H(v+\eta)=a(v) \\
& \left.W_{8}(-1,-1)\right|_{1,1}=\left.W_{8}(1,1)\right|_{-1,-1}=\Theta(2 \eta) H(v-\eta) \Theta(v+\eta)=b(v) \\
& \left.W_{8}(-1,1)\right|_{1,-1}=\left.W_{8}(1,-1)\right|_{-1,1}=H(2 \eta) \Theta(v-\eta) \Theta(v+\eta)=c(v) \\
& \left.W_{8}(1,-1)\right|_{1,-1}=\left.W_{8}(-1,1)\right|_{-1,1}=H(2 \eta) H(v-\eta) H(v+\eta)=d(v)
\end{aligned}
$$

The definition and useful properties of $H(v)$ and $\Theta(v)$ are recalled in the appendix.

In ref. [1] the matrix $Q_{R}(v)$ is defined as

$$
\left[Q_{R}(v)\right]_{\alpha \mid \beta}=\operatorname{Tr} S_{R}\left(\alpha_{1}, \beta_{1}\right) S_{R}\left(\alpha_{2}, \beta_{2}\right) \cdots S_{R}\left(\alpha_{N}, \beta_{N}\right)
$$

where $\alpha_{j}$ and $\beta_{j}= \pm 1$ and $S(\alpha, \beta)$ is an $L \times L$ matrix given as

$$
S_{R}(\alpha, \beta)=\left(\begin{array}{ccccccc}
z_{0} z_{-1} & 0 & 0 & \cdot & 0 \\
z_{1} & 0 & z_{-2} & 0 & . & 0 \\
0 & z_{2} & 0 & z_{-3} & \cdot & 0 \\
\cdot & \cdot & \cdot & \cdot & . & \cdot \\
0 & 0 & 0 & . & 0 & z_{1-L} \\
0 & 0 & 0 & \cdot & z_{L-1} & z_{L}
\end{array}\right)
$$

where

$$
z_{m}=q(\alpha, \beta, m \mid v)
$$

with

$$
\begin{aligned}
& q(+, \beta, m \mid v)=H(v+K+2 m \eta) \tau_{\beta, m}, \\
& q(-, \beta, m \mid v)=\Theta(v+K+2 m \eta) \tau_{\beta, m}
\end{aligned}
$$


and the integer $L$ is defined by (1.1). The $\tau_{\beta, m}$ are generically arbitrary but we note that if they are all set equal to unity then $Q_{R}(v)$ is so singular that its rank becomes 1 .

Furthermore ref. [1] also defines the companion matrix $Q_{L}(v)$

$$
\left[Q_{L}(v)\right]_{\alpha \mid \beta}=\operatorname{Tr} S_{L}\left(\alpha_{1}, \beta_{1}\right) S_{L}\left(\alpha_{2}, \beta_{2}\right) \cdots S_{L}\left(\alpha_{N}, \beta_{N}\right)
$$

where $\alpha_{j}$ and $\beta_{j}= \pm 1$ and $S_{L}(\alpha, \beta)$ is an $L \times L$ matrix given as

$$
S_{L}(\alpha, \beta)=\left(\begin{array}{cccccc}
z_{0}^{\prime} & z_{-1}^{\prime} & 0 & 0 & \cdot & 0 \\
z_{1}^{\prime} & 0 & z_{-2}^{\prime} & 0 & \cdot & 0 \\
0 & z_{2}^{\prime} & 0 & z_{-3}^{\prime} & \cdot & 0 \\
\cdot & \cdot & \cdot & \cdot & . & \cdot \\
0 & 0 & 0 & \cdot & 0 & z_{1-L}^{\prime} \\
0 & 0 & 0 & \cdot & z_{L-1}^{\prime} & z_{L}^{\prime}
\end{array}\right)
$$

with

$$
z_{m}^{\prime}=q^{\prime}(\alpha, \beta, m \mid v)
$$

and

$$
\begin{aligned}
& q(\alpha,+, m \mid v)=\tau_{\alpha, m}^{\prime} H(v-K-2 m \eta), \\
& q(\alpha,-, m \mid v)=\tau_{\alpha, m}^{\prime} \Theta(v-K-2 m \eta)
\end{aligned}
$$

In ref. [1] it is shown that $(\mathrm{C} 22)$

$$
T(v) Q_{R}(v)=h^{N}(v-\eta) Q_{R}(v+2 \eta)+h^{N}(v+\eta) Q_{R}(v-2 \eta)
$$

and $(\mathrm{C} 24)$

$$
Q_{L}(v) T(v)=h^{N}(v-\eta) Q_{L}(v+2 \eta)+h^{N}(v+\eta) Q_{L}(v-2 \eta)
$$

where

$$
h(v)=\Theta(0) \Theta(v) H(v)
$$

and it is further shown that $(\mathrm{C} 28)$

$$
Q_{L}(v) Q_{R}\left(v^{\prime}\right)=Q_{L}\left(v^{\prime}\right) Q_{R}(v) .
$$

Thus it follows that if we define (C37)

$$
Q_{72}(v)=Q_{R}(v) Q_{R}^{-1}\left(v_{0}\right)=Q_{L}^{-1}\left(v_{0}\right) Q_{L}(v)
$$


then (C38)

$$
T(v) Q_{72}(v)=h^{N}(v-\eta) Q_{72}(v+2 \eta)+h^{N}(v+\eta) Q_{72}(v-2 \eta)
$$

with

$$
\left[T(v), T\left(v^{\prime}\right)\right]=\left[T(v), Q_{72}\left(v^{\prime}\right)\right]=\left[Q_{72}(v), Q_{72}\left(v^{\prime}\right)\right]=0
$$

We note the periodicity relations

$$
\begin{aligned}
T(v \pm 2 K) & =(-1)^{N} T(v) \\
Q_{R, L}(v \pm 2 K) & =S Q_{R, L}(v)=Q_{R, L}(v) S \\
h(v+2 K) & =-h(v)
\end{aligned}
$$

where

$$
S=\prod_{j=1}^{N} \sigma_{j}^{z}
$$

which are consistent with (2.11) and (2.12) and we also note the commutation relation

$$
Q_{R}\left(v_{1}\right) Q_{R}^{-1}\left(v_{2}\right) Q_{R}\left(v_{3}\right)=Q_{R}\left(v_{3}\right) Q_{R}^{-1}\left(v_{2}\right) Q_{R}\left(v_{1}\right)
$$

\section{$\S 3 . \quad$ Fusion Relations and the Conjectured Functional Equation for $Q$}

The functional equation (2.16) is derived for the matrices $T(v)$ and $Q_{72}(v)$ but it follows from the commutation relations (2.17) that the four matrices $T(v), Q_{72}(v), Q_{72}(v \pm 2 \eta)$ may be simultaneously diagonalised and thus (2.16) may be regarded as an equation for the eigenvalues. The matrix $Q_{72}(v)$ is found (empirically, we know of no mathematical proof in the literature) to be nondegenerate and thus if $T(v)$ were also nondegenerate there would be a $1-1$ map between eigenvalues of $T(v)$ and $Q_{72}(v)$. However, in ref. [1] the condition (1.1) holds and in this case, when the number of sites in the lattice $N$ is sufficiently large, the matrix $T(v)$ always has degenerate eigenvalues. There is thus a many to one map of eigenvalues of $Q_{72}(v)$ to eigenvalues of $T(v)$ and this leads to the fact that the functional equation is not sufficient to determine all the eigenvalues of $Q_{72}(v)$ which correspond to a degenerate eigenvalue of $T(v)$.

In order to resolve this problem of degeneracy and multiplicity of the eigenvalue of $T(v)$ we recently conjectured [10] the following functional equation for $Q_{72}$. 
Conjecture.

For either $L$ even or $L$ and $m_{1}$ odd

$$
\begin{aligned}
& e^{-N \pi i v / 2 K} Q_{72}\left(v-i K^{\prime}\right) \\
& =A \sum_{l=0}^{L-1} h^{N}\left(v-(2 l+1) m_{1} K / L\right) \\
& \quad \times \frac{Q_{72}(v)}{Q_{72}\left(v-2 l m_{1} K / L\right) Q_{72}\left(v-2(l+1) m_{1} K / L\right)}
\end{aligned}
$$

where $A$ is a normalizing constant matrix independent of $v$ that commutes with $Q_{72}(v)$. What this matrix is depends on the normalization value of $v_{0}$ in the definition (2.15) of $Q_{72}(v)$.

We prove this conjecture for $L=2$ in Sec. 7 and have numerically verified it for $L=3$ for various values of $N$.

The matrix $Q_{72}(v)$ as defined by $(2.15)$ is not in the form of $Q_{R}(v)$ and $Q_{L}(v)$ of being the trace of a product of matrices and thus it is natural to rewrite the conjecture (3.1) in terms of $Q_{L}(v)$ and $Q_{R}(v)$ as

$$
\begin{aligned}
& e^{-N \pi i v / 2 K} Q_{L}\left(v-i K^{\prime}\right)=Q_{L}\left(v_{0}\right) A Q_{R}\left(v_{0}\right) \\
& \quad \times \sum_{l=0}^{L-1} h^{N}\left(v-(2 l+1) m_{1} K / L\right) \\
& \quad \times Q_{R}^{-1}\left(v-2 l m_{1} K / L\right) Q_{R}(v) Q_{R}^{-1}\left(v-2(l+1) m_{1} K / L\right)
\end{aligned}
$$

The form (3.2) of the conjecture is strikingly similar in form to the functional equation (4.40) in ref. [6] of the chiral Potts model if we make the identification of $Q_{L}(v)$ with $\hat{T}_{c p}$ and $Q_{R}(v)$ with $T_{c p}$ where the subscript cp indicates the quantities in ref. [6]. It is therefore natural to search for a proof of (3.1) by following the methods of ref. [6].

We begin by writing (2.11) in the form

$$
T(v)=h^{N}(v-\eta) Q_{R}(v+2 \eta) Q_{R}^{-1}(v)+h^{N}(v+\eta) Q_{R}(v-2 \eta) Q_{R}^{-1}(v)
$$

which is analogous to (4.20) of ref. [6] if we identify $T(v)$ with $\tau_{c p}^{(2)}$.

To continue we need to define quantities analogous to $\tau_{c p}^{(j)}$ which obey functional equations analogous to (4.27a) of ref. [6]. The appropriate objects are the "fusion matrices" $T^{(j)}(v)$ which may be defined recursively, for any $\eta$ not just (1.1), by 


$$
\begin{aligned}
& T^{(2)}(v)=T(v) \\
& \begin{aligned}
T^{(2)}(v-2 \eta) T^{(2)}(v-4 \eta)= & h^{N}(v-3 \eta) T^{(3)}(v-4 \eta) \\
& +h^{N}(v-\eta) h^{N}(v-5 \eta)
\end{aligned}
\end{aligned}
$$

and for $j \geq 3$

$$
\begin{aligned}
& T^{(2)}(v-2 \eta) T^{(j)}(v-2 j \eta) \\
& \quad=h^{N}(v-3 \eta) T^{(j+1)}(v-2 j \eta)+h^{N}(v-\eta) T^{(j-1)}(v-2 j \eta) .
\end{aligned}
$$

From these defining equations we show that $T^{(j)}$ may be written in terms of $Q_{R}(v)$ as

$$
\begin{aligned}
& T^{(j)}\left(v-2(j-1) m_{1} K / L\right)=\sum_{l=0}^{j-1} h^{N}\left(v-(2 l+1) m_{1} K / L\right) \\
& \quad \times Q_{R}(v) Q_{R}^{-1}\left(v-2 l m_{1} K / L\right) Q_{R}\left(v-2 j m_{1} K / L\right) Q_{R}^{-1}\left(v-2(l+1) m_{1} K / L\right)
\end{aligned}
$$

by directly substituting (3.7) into (3.5) and (3.6), using the commutation relation (2.22) and noting that (3.7) reduces to (3.3) if we set $j=2$ and send $v \rightarrow v+2 \eta$. Equation (3.7) is the analogue of (4.34) of ref. [6].

Now define

$$
M=Q_{L}\left(v_{0}\right) A Q_{R}\left(v_{0}\right)
$$

and multiply (3.2) on the left by $Q_{R}(v) M^{-1}$ we obtain

$$
\begin{aligned}
& e^{-N \pi i v / 2 K} Q_{R}(v) M^{-1} Q_{L}\left(v-i K^{\prime}\right) \\
& =\sum_{l=0}^{L-1} h^{N}\left(v-(2 l+1) m_{1} K / L\right) Q_{R}(v) \\
& \quad \times Q_{R}^{-1}\left(v-2 l m_{1} K / L\right) Q_{R}(v) Q_{R}^{-1}\left(v-2(l+1) m_{1} K / L\right)
\end{aligned}
$$

If we now use the periodicity property (2.19) in the right hand side of (3.7) with $j=L$ and compare with the right hand side of the conjectured functional equation (3.9) we conclude that the conjectured functional equation will hold if we can prove that

$$
\begin{aligned}
& T^{(L)}\left(v-2(L-1) m_{1} K / L\right) \\
& \quad=e^{-i N \pi v / 2 K} Q_{R}(v) M^{-1} Q_{L}\left(v-i K^{\prime}\right) S^{m_{1}} .
\end{aligned}
$$

which is the analogue of (4.44) of ref. [6].

We conclude this section by noting that in the chiral Potts model there is a functional equation $(4.27 \mathrm{c})$ of ref. [6] which relates $\tau^{(L+1)}$ to $\tau^{(L-1)}$. To 
obtain the analogous equation in the eight vertex model we specialize $j=L+1$ in (3.7) and use (2.19) to get

$$
\begin{aligned}
& T^{(L+1)}\left(v-2 m_{1} K\right)=\sum_{l=0}^{L} h^{N}\left(v-(2 l+1) m_{1} K / L\right) \\
& \quad \times Q_{R}(v) Q_{R}^{-1}\left(v-2 l m_{1} K / L\right) Q_{R}\left(v-2 m_{1} K / L\right) S^{m_{1}} Q_{R}^{-1}\left(v-2(l+1) m_{1} K / L\right)
\end{aligned}
$$

We now write the terms $l=0, L$ separately and use (2.20) to find

$$
\begin{aligned}
& T^{(L+1)}\left(v-2 m_{1} K\right) \\
& =\left[1+(-1)^{N}\right] h^{N}\left(v-m_{1} K / L\right) S^{m_{1}}+\sum_{l=1}^{L-1} h^{N}\left(v-(2 l+1) m_{1} K / L\right) \\
& \times Q_{R}(v) Q_{R}^{-1}\left(v-2 l m_{1} K / L\right) Q_{R}\left(v-2 m_{1} K / L\right) S^{m_{1}} Q_{R}^{-1}\left(v-2(l+1) m_{1} K / L\right) .
\end{aligned}
$$

In the sum we use the commutation relation (2.22), set $l=k+1$ and use (2.19) to write $S^{m_{1}} Q_{R}(v)=Q_{R}\left(v-2 m_{1} K\right)=Q_{R}\left(v-2 m_{1} K / L-2(L-1) m_{1} K / L\right)$ and thus we find

$$
\begin{aligned}
& T^{(L+1)}\left(v-2 m_{1} K\right) \\
& =\left[1+(-1)^{N}\right] h^{N}\left(v-m_{1} K / L\right) S^{m_{1}}+\sum_{k=0}^{L-2} h^{N}\left(v-2 m_{1} K / L-(2 k+1) m_{1} K / L\right) \\
& \quad \times Q_{R}\left(v-2 m_{1} K / L\right) Q_{R}^{-1}\left(v-2 m_{1} K / L-2 k m_{1} K / L\right) \\
& \quad \times Q_{R}\left(v-2 m_{1} K / L-2(L-1) m_{1} K / L\right) \\
& \quad \times Q_{R}^{-1}\left(v-2 m_{1} K / L-2(k+1) m_{1} K / L\right) .
\end{aligned}
$$

The sum on the right hand side is seen to be $T^{(L-1)}\left(v-2 m_{1} K / L-2(L-\right.$ 2) $\left.m_{1} K / L\right)$ by use of (3.7) and thus

$$
\begin{aligned}
T^{(L+1)}\left(v-2 m_{1} K\right)= & {\left[1+(-1)^{N}\right] h^{N}\left(v-m_{1} K / L\right) S^{m_{1}} } \\
& +T^{(L-1)}\left(v+2 m_{1} K / L-2 m_{1} K\right)
\end{aligned}
$$

and finally by use of (2.18) we obtain the desired result

$$
\begin{aligned}
T^{(L+1)}(v)= & {\left[1+(-1)^{N}\right] h^{N}\left(v-m_{1} K / L\right) S^{m_{1}} } \\
& +T^{(L-1)}\left(v+2 m_{1} K / L\right)
\end{aligned}
$$


In the six vertex limit this functional equation was first exhibited by Nepomechie as (1.3) of ref. [18] where it is proven for $L=2,3,4$ and in (2.17) in ref. [19] and it was used to study various open six vertex chains at roots of unity in [18-20]. For the eight vertex model this result has been obtained by Bazhanov and Stroganov (see footnote $\mathrm{g}$ on page 141 of [21]) and Baxter $[22]$.

\section{§4. Explicit Fusion Matrices for $J=3,4,5$}

We introduced the matrices $T^{(j)}(v)$ by the recursion relations (3.4)-(3.6) and called them "fusion matrices". However in ref. [6] the analogous matrices $\tau^{(j)}$ are not defined by the analogue of (3.4)-(3.6) but rather they are defined as the trace of products of explicitly given $j \times j$ matrices and (3.4)-(3.6) follows as a theorem. The explicit form of $\tau^{(j)}$ is used in the proof of the analogue of (3.10).

The existence of matrices $T^{(j)}(v)$ written in the form

$$
\left.T^{(j)}(v)\right|_{\mu, \nu}=\operatorname{Tr} R^{(j)}\left(\mu_{1}, \nu_{2}\right)(v) \cdots R^{(j)}\left(\mu_{N}, \nu_{N}\right)(v)
$$

with $R^{(j)}(\mu, \nu)$ being $j \times j$ matrices which generalizes (2.1) and has the global property that $\left[T^{(j)}(v), T^{(j)}\left(v^{\prime}\right)\right]=0$ by virtue of $R^{(j)}(\mu, \nu)(v)$ and $R^{(j)}(\mu, \nu)\left(v^{\prime}\right)$ satisfying a local Yang-Baxter equation using

$$
R^{(2)}(\mu, \nu)(v)=W_{8}(\mu, \nu)
$$

as the elements of the $2 \times 2$ intertwining matrix has been extensively studied [11-17] for both the 8 and the 6 vertex model. The final result of these studies for the 8 vertex model is given by lemma 2.3.1 of ref. [17] which says that $T^{(j)}(v)$ as defined by (4.1) is given in terms of a matrix $R^{(j)}(\mu, \nu)(v)$ which is constructed from $R^{(2)}(\mu, \nu)(v)$ by

$$
\begin{aligned}
& R^{(j)}(\mu, \nu)(v)= \\
& \frac{P \sum_{\nu_{1}, \cdots \nu_{j-2}} R^{(2)}\left(\mu, \nu_{1}\right)(v) R^{(2)}\left(\nu_{1}, \nu_{2}\right)(v+2 \eta) \cdots R^{(2)}\left(\nu_{j-2}, \mu\right)(v+2(j-2) \eta)}{\prod_{l=0}^{j-3} h[v+(2 l+1) \eta]}
\end{aligned}
$$

where $P$ is the projection from the internal space of dimension $2^{j-1}$ to the space of dimension $j$ of completely symmetric tensors. This construction is known as "fusion". The matrix $R^{(j)}(v)$ has no poles and $T^{(j)}(v)$ satisfies (3.4)-(3.6). 
Unfortunately the result (4.3) is not as explicit as the expression for $\tau^{(j)}$ in the chiral Potts model [6] or the corresponding expression for the fusion weights in the RSOS model [17]. However, it would appear that such an explicit form would be of help in proving the conjecture (3.10) for the 8 vertex model. Therefore in order to gain insight into the fusion matrices we have constructed the matrices $T^{(j)}(v)$ directly from (4.3) for $j=3,4,5$. The computation is straightforward and makes extensive use of properties of theta functions (presented in detail for example in ref. [23]). In particular we use the two addition formulas for theta functions (15.4.25) and (15.4.26) of ref. [24].

$$
\begin{aligned}
& \Theta(u) \Theta(v) \Theta(a-u) \Theta(a-v)-H(u) H(v) H(a-u) H(a-v) \\
& =\Theta(0) \Theta(a) \Theta(u-v) \Theta(a-u-v)
\end{aligned}
$$

and

$$
\begin{aligned}
& H(v) H(a-v) \Theta(u) \Theta(a-u)-\Theta(v) \Theta(a-v) H(u) H(a-u) \\
& =\Theta(0) \Theta(a) H(v-u) H(a-u-v)
\end{aligned}
$$

and the fact that in the set of functions given for $a$ fixed and $j$ an integer

$$
\Theta(v+j \eta) H(v+(a-j) \eta)
$$

only two are linearly independent. Similarly of the functions for fixed $a$

$$
\Theta(v+j \eta) \Theta(v+(a-j) \eta), \quad H(v+j \eta) H(v+(a-j) \eta)
$$

only two are linearly independent. There are accordingly many equivalent ways to write the theta functions in $R^{(j)}(\mu, \nu)(v)$.

We note that the matrix $R^{(j)}(-,-)(v)$ is obtained from $R^{(j)}(+,+)(v)$ and the matrix $R^{(j)}(-,+)(v)$ is obtained from $R^{(j)}(+,-)(v)$ by the interchange $\Theta(v+2 k \eta) \leftrightarrow H(v+2 k \eta)$. Furthermore the matrix elements have the symmetry property

$$
\begin{aligned}
& R^{(j)}(\mu, \nu)(v)_{m, n} \leftrightarrow R^{(j)}(\mu, \nu)(v)_{j-1-m, j-1-n} \\
& \text { by the substitution } \Theta(v+2 a \eta) \leftrightarrow H(v+2 a \eta)
\end{aligned}
$$

With these provisos we have the following results: 


\section{A. The matrices $R^{(3)}(\mu, \nu)(v)$}

The matrix $R^{(3)}(+,+)(v)$ is

$$
\begin{aligned}
& R^{(3)}(+,+)(v) \\
& =\left(\begin{array}{ccc}
\frac{\Theta^{2}(2 \eta)}{\Theta(0)} \Theta(v-\eta) & & \frac{H^{2}(2 \eta)}{\Theta(0)} H(v-\eta) \\
\times H(v+3 \eta) & 0 & \times \Theta(v+3 \eta) \\
0 & \Theta(4 \eta) H(v+\eta) \Theta(v+\eta) & 0 \\
\frac{H^{2}(2 \eta)}{\Theta(0)} \Theta(v-\eta) & 0 & \frac{\Theta^{2}(2 \eta)}{\Theta(0)} H(v-\eta) \\
\times H(v+3 \eta) & & \times \Theta(v+3 \eta)
\end{array}\right)
\end{aligned}
$$

and the matrix $R^{(3)}(+,-)(v)$ is

$$
\begin{aligned}
& R^{(3)}(+,-)(v) \\
& =\left(\begin{array}{ccc}
0 & H(4 \eta) H^{2}(v+\eta) & 0 \\
\frac{\Theta(2 \eta) H(2 \eta)}{\Theta(0)} \Theta(v-\eta) & & \frac{H(2 \eta) \Theta(2 \eta)}{\Theta(0)} H(v-\eta) \\
\times \Theta(v+3 \eta) & 0 & \times H(v+3 \eta) \\
0 & H(4 \eta) \Theta^{2}(v+\eta) & 0
\end{array}\right) .
\end{aligned}
$$

B. The matrices $R^{(4)}(\mu, \nu)(v)$

The matrix $R^{(4)}(+,+)(v)$ is

$$
R^{(4)}(+,+)(v)=\left(\begin{array}{cccc}
R_{00}^{(4)} & 0 & R_{02}^{(4)} & 0 \\
0 & R_{11}^{(4)} & 0 & R_{13}^{(4)} \\
R_{20}^{(4)} & 0 & R_{22}^{(4)} & 0 \\
0 & R_{31}^{(4)} & 0 & R_{33}^{(4)}
\end{array}\right)
$$

where the 4 independent elements of $R^{(4)}(++)$ are

$$
\begin{aligned}
& R_{00}^{(4)}(+,+)=\frac{\Theta^{3}(2 \eta)}{\Theta^{2}(0)} \Theta(v-\eta) H(v+5 \eta) \\
& R_{20}^{(4)}(+,+)=\frac{H^{2}(2 \eta) \Theta(2 \eta)}{\Theta^{2}(0)} \Theta(v-\eta) H(v+5 \eta)
\end{aligned}
$$




$$
\begin{aligned}
R_{11}^{(4)}(+,+)= & \frac{\Theta(4 \eta) H(4 \eta)}{H(2 \eta)} H(v+\eta) \Theta(v+3 \eta) \\
& -\frac{\Theta^{3}(2 \eta)}{\Theta^{2}(0)} H(v-\eta) \Theta(v+5 \eta) \\
R_{31}^{(4)}(+,+)= & \frac{H^{2}(4 \eta)}{\Theta(2 \eta)} \Theta(v+\eta) H(v+3 \eta) \\
& -\frac{H^{2}(2 \eta) \Theta(2 \eta)}{\Theta^{2}(0)} H(v-\eta) \Theta(v+5 \eta)
\end{aligned}
$$

and the remaining 4 elements are obtained by the symmetry (4.8).

The matrix $R^{(4)}(+,-)(v)$ is

$$
R^{(4)}(+,-)(v)=\left(\begin{array}{cccc}
0 & R_{01}^{(4)} & 0 & R_{03}^{(4)} \\
R_{10}^{(4)} & 0 & R_{12}^{(4)} & 0 \\
0 & R_{21}^{(4)} & 0 & R_{23}^{(4)} \\
R_{30}^{(4)} & 0 & R_{32}^{(4)} & 0
\end{array}\right)
$$

where the 4 independent elements are

$$
\begin{aligned}
R_{10}^{(4)}(+,-)= & \frac{\Theta^{2}(2 \eta) H(2 \eta)}{\Theta^{2}(0)} \Theta(v-\eta) \Theta(v+5 \eta) \\
R_{30}^{(4)}(+,-)= & \frac{H^{3}(2 \eta)}{\Theta^{2}(0)} \Theta(v-\eta) \Theta(v+5 \eta) \\
R_{21}^{(4)}(+,-)= & -\frac{H^{3}(2 \eta) \Theta(4 \eta)}{\Theta^{3}(0)} H(v+\eta) H(v+3 \eta) \\
& +\frac{\Theta^{3}(2 \eta) H(4 \eta)}{\Theta^{3}(0)} \Theta(v+\eta) \Theta(v+3 \eta) \\
= & \frac{\Theta^{2}(4 \eta) H(4 \eta)}{\Theta(0) \Theta(6 \eta)} \Theta(v-\eta) \Theta(v+5 \eta) \\
& +\frac{\Theta(2 \eta) H^{2}(2 \eta) H(6 \eta)}{\Theta^{2}(0) \Theta(6 \eta)} H(v-\eta) H(v+5 \eta) \\
& -\frac{\Theta^{2}(2 \eta) H(2 \eta)}{\Theta^{2}(0)} H(v-\eta) H(v+5 \eta) \\
& +\frac{H^{2}(4 \eta)}{H(2 \eta)} H(v+\eta) H(v+3 \eta)
\end{aligned}
$$




$$
\begin{aligned}
= & \frac{H^{3}(4 \eta)}{\Theta(0) \Theta(6 \eta)} \Theta(v-\eta) \Theta(v+5 \eta) \\
& +\frac{\Theta^{3}(2 \eta) H(6 \eta)}{\Theta^{2}(0) \Theta(6 \eta)} H(v-\eta) H(v+5 \eta)
\end{aligned}
$$

and the four other elements obtained by the symmetry (4.8).

\section{The matrices $R^{(5)}(\mu . \nu)(v)$}

The matrix $R^{(5)}(+,+)(v)$ is

$$
R^{(5)}(+,+)(v)=\left(\begin{array}{ccccc}
R_{00}^{(5)} & 0 & R_{02}^{(5)} & 0 & R_{04}^{(5)} \\
0 & R_{11}^{(5)} & 0 & R_{13}^{(5)} & 0 \\
R_{20}^{(5)} & 0 & R_{22}^{(5)} & 0 & R_{24}^{(5)} \\
0 & R_{31}^{(5)} & 0 & R_{33}^{(5)} & 0 \\
R_{40}^{(5)} & 0 & R_{42}^{(5)} & 0 & R_{44}^{(5)}
\end{array}\right)
$$

where the 6 independent non symmetric elements are

$$
\begin{aligned}
R_{00}^{(5)}(+,+)= & \frac{\Theta^{4}(2 \eta)}{\Theta^{3}(0)} \Theta(v-\eta) H(v+7 \eta) \\
R_{20}^{(5)}(+,+)= & \frac{H^{2}(2 \eta) \Theta^{2}(2 \eta)}{\Theta^{3}(0)} \Theta(v-\eta) H(v+7 \eta) \\
R_{40}^{(5)}(+,+)= & \frac{H^{4}(2 \eta)}{\Theta^{3}(0)} \Theta(v-\eta) H(v+7 \eta) \\
R_{11}^{(5)}(+,+)= & \frac{\Theta^{2}(2 \eta) \Theta^{2}(4 \eta)}{\Theta^{3}(0)} \Theta(v+\eta) H(v+5 \eta) \\
& -\frac{H^{2}(2 \eta) H^{2}(4 \eta)}{\Theta^{3}(0)} H(v+\eta) \Theta(v+5 \eta) \\
R_{31}^{(5)}(++)= & \frac{\Theta^{2}(2 \eta) H^{2}(4 \eta)}{\Theta^{3}(0)} \Theta(v+\eta) H(v+5 \eta) \\
& -\frac{H^{2}(2 \eta) \Theta^{2}(4 \eta)}{\Theta^{3}(0)} H(v+\eta) \Theta(v+5 \eta) \\
R_{02}^{(5)}(+,+)= & -\frac{H^{2}(2 \eta) \Theta^{2}(2 \eta) H(8 \eta)}{\Theta^{2}(0) H(4 \eta) \Theta(4 \eta)} H(v+3 \eta) \Theta(v+3 \eta) \\
& +\frac{H^{3}(4 \eta)}{H(2 \eta) \Theta(2 \eta)} H(v+\eta) \Theta(v+5 \eta),
\end{aligned}
$$


the companion non symmetric elements are obtained by the symmetry (4.8) and the one symmetric element is

$$
\begin{aligned}
R_{22}^{(5)}(+,+)= & \Theta^{-3}(0)\left(\Theta^{3}(2 \eta) \Theta(6 \eta)\right. \\
& \left.-H^{3}(2 \eta) H(6 \eta)\right) H(v+3 \eta) \Theta(v+3 \eta)
\end{aligned}
$$

The matrix $R^{(5)}(+,-)(v)$ is

$$
R^{(5)}(+,-)(v)=\left(\begin{array}{ccccc}
0 & R_{01}^{(5)} & 0 & R_{03}^{(5)} & 0 \\
R_{10}^{(5)} & 0 & R_{12}^{(5)} & 0 & R_{14}^{(5)} \\
0 & R_{21}^{(5)} & 0 & R_{23}^{(5)} & 0 \\
R_{30}^{(5)} & 0 & R_{32}^{(5)} & 0 & R_{34}^{(5)} \\
0 & R_{41}^{(5)} & 0 & R_{43}^{(5)} & 0
\end{array}\right)
$$

where the six independent elements are

$$
\begin{aligned}
R_{10}^{(5)}(+,-)= & \frac{H(2 \eta) \Theta^{3}(2 \eta)}{\Theta^{3}(0)} \Theta(v-\eta) \Theta(v+7 \eta) \\
R_{30}^{(5)}(+,-)= & \frac{H^{3}(2 \eta) \Theta(2 \eta)}{\Theta^{3}(0)} \Theta(v-\eta) \Theta(v+7 \eta) \\
R_{01}^{(5)}(+,-)= & \frac{\Theta(2 \eta)}{\Theta^{2}(0) \Theta(6 \eta)}\left[H^{3}(4 \eta) \Theta(v-\eta) \Theta(v+7 \eta)\right. \\
& \left.+\Theta^{2}(2 \eta) H(8 \eta) H(v+\eta) H(v+5 \eta)\right] \\
R_{21}^{(5)}(+,-)= & \frac{H(4 \eta) \Theta(4 \eta)}{\Theta^{3}(0)}\left[\Theta^{2}(2 \eta) \Theta(v+\eta) \Theta(v+5 \eta)\right. \\
& \left.-H^{2}(2 \eta) H(v+\eta) H(v+5 \eta)\right] \\
R_{41}^{(5)}(+,-)= & \frac{H(2 \eta)}{\Theta^{2}(0) H(6 \eta)}\left[H^{3}(4 \eta) \Theta(v-\eta) \Theta(v+7 \eta)\right. \\
& \left.+H^{2}(2 \eta) H(8 \eta) \Theta(v+\eta) \Theta(v+5 \eta)\right] \\
R_{12}^{(5)}(+,-)= & \frac{H(2 \eta) \Theta(6 \eta)}{\Theta(0)} H(v+\eta) H(v+5 \eta) \\
& +\frac{H(2 \eta) \Theta^{3}(2 \eta) H(8 \eta)}{\Theta^{2}(0) H(4 \eta) \Theta(4 \eta)} H^{2}(v+3 \eta)
\end{aligned}
$$

and the other six elements are obtained by the symmetry (4.8).

\section{$\S 5 . \quad$ A Similarity Transformation}

The fusion matrices derived in the preceding section by direct application of the fusion construction do not have a particularly revealing form. Furthermore 
they do not directly reveal the reduction (3.15) of $T^{(L+1)}(v)$ at the root of unity point (1.1). However, because the form (4.1) for $T^{(j)}(v)$ is the trace of a product of matrices any similarity transformation of the $R^{(j)}(\mu, \nu)(v)$

$$
\tilde{R}^{(j)}(\mu, \nu)(v)=M_{j}(\eta) R^{(j)}(\mu, \nu)(v) M_{j}^{-1}(\eta)
$$

with $M_{j}(\eta)$ independent of $v$ is just as good for our purposes as the original $R^{(j)}(\mu, \nu)(v)$.

The form of the functional equation (3.15) will follow if we can determine a similarity transformation matrix $M_{j}(\eta)$ such that when the root of unity condition (1.1) holds the matrix elements of $\tilde{R}^{(L+1)}(\mu, \nu)$ have the property that

$$
\tilde{R}^{(L+1)}(\mu, \nu)_{0, k}=\tilde{R}^{(L+1)}(\mu, \nu)_{L-1, k}=0 \text { for } 1 \leq k \leq L-2
$$

The matrix $R^{(3)}(\mu, \nu)$ already has this property but the matrices $R^{(4)}(\mu, \nu)$ and $R^{(5)}(\mu, \nu)$ do not. However, because the functional equation (3.15) has been proven true in Sec. 3 by an independent method it must be possible to find a similarity transformation which does in fact put $R^{(4)}(\mu, \nu)$ and $R^{(5)}(\mu, \nu)$ into the required form. It is straightforward to determine these matrices. In fact these matrices are not unique and have several arbitrary parameters we can freely chose. We have determined the families of these similarity transformations for $L=3$ and 4 . These similarity transformations may then be extended from the root of unity case to the case of arbitrary $\eta$ essentially by replacing $m_{1} K / L$ everywhere by $\eta$. When this is done we find the following similarity transformations which are easily verified.

\section{A. Transformation of $R^{(4)}(\mu, \nu)$}

The transformation matrix is

$$
M_{4}(\eta)=\left(\begin{array}{cccc}
1 & 0 & -f & 0 \\
0 & 1 & 0 & 0 \\
0 & 0 & 1 & 0 \\
0 & -f & 0 & 1
\end{array}\right)
$$

and

$$
M_{4}^{-1}(\eta)=\left(\begin{array}{llll}
1 & 0 & f & 0 \\
0 & 1 & 0 & 0 \\
0 & 0 & 1 & 0 \\
0 & f & 0 & 1
\end{array}\right)
$$


with

$$
f=H^{2}(2 \eta) / \Theta^{2}(2 \eta)
$$

Thus using the notation

$$
\begin{aligned}
& \Theta_{a}=\Theta(v+a \eta) \\
& H_{a}=H(v+a \eta)
\end{aligned}
$$

we have

$$
\begin{aligned}
\tilde{R}^{(4)}(+,+)= & M_{4}(\eta) R^{(4)}(++) M_{4}^{-1}(\eta) \\
= & \left(\begin{array}{ccccc}
C_{00}^{(4)} \Theta_{-1} H_{5} & 0 & C_{02}^{(4)} \Theta_{3} H_{1} & 0 \\
0 & C_{11}^{(4)} \Theta_{1} H_{3} & 0 & C_{13}^{(4)} \Theta_{5} H_{-1} \\
C_{20}^{(4)} \Theta_{-1} H_{5} & 0 & C_{22}^{(4)} \Theta_{3} H_{1} & 0 \\
0 & C_{31}^{(4)} \Theta_{1} H_{3} & 0 & C_{33}^{(4)} \Theta_{5} H_{-1}
\end{array}\right)
\end{aligned}
$$

where

$$
\begin{aligned}
& C_{00}^{(4)}=C_{33}^{(4)}=\frac{\Theta(0) \Theta(4 \eta)}{\Theta(2 \eta)} \\
& C_{22}^{(4)}=C_{11}^{(4)}=\frac{\Theta^{2}(4 \eta)}{\Theta(2 \eta)} \\
& C_{20}^{(4)}=C_{13}^{(4)}=\frac{H^{2}(2 \eta) \Theta(2 \eta)}{\Theta^{2}(0)} \\
& C_{31}^{(4)}=C_{02}^{(4)}=\frac{\Theta^{2}(0) H(2 \eta) H(6 \eta)}{\Theta^{3}(2 \eta)}
\end{aligned}
$$

and

$$
\begin{aligned}
\tilde{R}^{(4)}(+,-) & =M_{4}(\eta) R^{(4)}(+,-) M_{4}^{-1}(\eta) \\
& =\left(\begin{array}{cccc}
0 & C_{01}^{(4)} H_{1} H_{3} & 0 & 0 \\
C_{10}^{(4)} \Theta_{-1} \Theta_{7} & 0 & C_{12}^{(4)} H_{1} H_{3} & 0 \\
0 & C_{21}^{(4)} \Theta_{1} \Theta_{3} & 0 & C_{23}^{(4)} H_{-1} H_{5} \\
0 & 0 & C_{32}^{(4)} \Theta_{1} \Theta_{3} & 0
\end{array}\right)
\end{aligned}
$$

with

$$
\begin{aligned}
& C_{10}^{(4)}=C_{23}^{(4)}=\frac{\Theta^{2}(2 \eta) H(2 \eta)}{\Theta^{2}(0)} \\
& C_{21}^{(4)}=C_{12}^{(4)}=\frac{H(4 \eta) \Theta(4 \eta)}{\Theta(2 \eta)} \\
& C_{32}^{(4)}=C_{01}^{(4)}=\frac{\Theta^{2}(0) H(6 \eta)}{\Theta^{2}(2 \eta)}
\end{aligned}
$$




\section{B. Transformation of $R^{(5)}(\mu, \nu)$}

The transformation matrix is

$$
M_{5}(\eta)=\left(\begin{array}{ccccc}
1 & 0 & f & 0 & 0 \\
0 & 1 & 0 & g & 0 \\
0 & 0 & h & 0 & 0 \\
0 & g & 0 & 1 & 0 \\
0 & 0 & f & 0 & 1
\end{array}\right)
$$

where

$$
\begin{aligned}
& f=-\frac{H^{2}(4 \eta)}{\Theta^{2}(4 \eta)} \\
& g=-\frac{H^{2}(2 \eta)}{\Theta^{2}(2 \eta)} \\
& h=\frac{\Theta^{2}(0) H(2 \eta)}{H(4 \eta) \Theta(4 \eta) \Theta(2 \eta)}
\end{aligned}
$$

and

$$
M_{5}^{-1}(\eta)=\left(\begin{array}{ccccc}
1 & 0 & a & 0 & 0 \\
0 & b & 0 & c & 0 \\
0 & 0 & d & 0 & 0 \\
0 & c & 0 & b & 0 \\
0 & 0 & a & 0 & 1
\end{array}\right)
$$

with

$$
\begin{aligned}
& a=-\frac{f}{h}=\frac{H^{3}(4 \eta) \Theta(2 \eta)}{\Theta(4 \eta) \Theta^{2}(0) H(2 \eta)} \\
& b=\frac{1}{1-g^{2}}=\frac{\Theta^{4}(2 \eta)}{\Theta^{3}(0) \Theta(4 \eta)} \\
& c=-\frac{g}{1-g^{2}}=\frac{H^{2}(2 \eta) \Theta^{2}(2 \eta)}{\Theta^{3}(0) \Theta(4 \eta)} \\
& d=\frac{1}{h}=\frac{H(4 \eta) \Theta(4 \eta) \Theta(2 \eta)}{H(2 \eta) \Theta^{2}(0)}
\end{aligned}
$$


Thus we find

$$
\begin{aligned}
\tilde{R}^{(5)}(+,+)= & M_{5}(\eta) R^{(5)}(++) M_{5}(\eta)^{-1} \\
& \times\left(\begin{array}{cccccc}
C_{00}^{(5)} \Theta_{-1} H_{7} & 0 & C_{02}^{(5)} \Theta_{3} H_{3} & 0 & C_{04}^{(5)} \Theta_{7} H_{-1} \\
0 & C_{11}^{(5)} \Theta_{1} H_{5} & 0 & C_{13}^{(5)} \Theta_{5} H_{1} & 0 \\
C_{20}^{(5)} \Theta_{-1} H_{7} & 0 & C_{22}^{(5)} \Theta_{3} H_{3} & 0 & C_{24}^{(5)} \Theta_{7} H_{-1} \\
0 & C_{31}^{(5)} \Theta_{1} H_{5} & 0 & C_{33}^{(5)} \Theta_{5} H_{1} & 0 \\
C_{40}^{(5)} \Theta_{-1} H_{7} & 0 & C_{42}^{(5)} \Theta_{3} H_{3} & 0 & C_{44}^{(5)} \Theta_{7} H_{-1}
\end{array}\right)
\end{aligned}
$$

where

$$
\begin{aligned}
& C_{00}^{(5)}=C_{44}^{(5)}=\frac{\Theta^{3}(2 \eta) \Theta(6 \eta)}{\Theta(0) \Theta^{2}(4 \eta)} \\
& C_{20}^{(5)}=C_{24}^{(5)}=\frac{H^{3}(2 \eta) \Theta(2 \eta)}{\Theta(0) \Theta(4 \eta) H(4 \eta)} \\
& C_{40}^{(5)}=C_{04}^{(5)}=\frac{H^{3}(2 \eta) H(6 \eta)}{\Theta(0) \Theta^{2}(4 \eta)} \\
& C_{02}^{(5)}=C_{42}^{(5)}=\frac{H(8 \eta) H(6 \eta) \Theta(6 \eta) \Theta^{2}(2 \eta)}{\Theta^{4}(4 \eta)} \\
& C_{11}^{(5)}=C_{33}^{(5)}=\frac{\Theta(2 \eta) \Theta(6 \eta)}{\Theta(0)} \\
& C_{13}^{(5)}=C_{31}^{(5)}=\frac{H(2 \eta) H(6 \eta)}{\Theta(0)} \\
& C_{22}^{(5)}=\frac{1}{\Theta(0) \Theta^{2}(4 \eta)}\left(\Theta^{2}(2 \eta) \Theta^{2}(6 \eta)+H^{2}(2 \eta) H^{2}(6 \eta)\right)
\end{aligned}
$$

and

$$
\begin{aligned}
\tilde{R}^{(5)}(+,-) & =M_{5}(\eta) R^{(5)}(+-) M_{5}(\eta)^{-1} \\
& =\left(\begin{array}{cccccc}
0 & C_{01}^{(5)} H_{1} H_{5} & 0 & 0 & 0 \\
C_{10}^{(5)} \Theta_{-1} \Theta_{7} & 0 & C_{12}^{(5)} H_{3}^{2} & 0 & 0 \\
0 & C_{21}^{(5)} \Theta_{1} \Theta_{5} & 0 & C_{23}^{(5)} H_{1} H_{5} & 0 \\
0 & 0 & C_{32}^{(5)} \Theta_{3}^{2} & 0 & C_{34}^{(5)} H_{-1} H_{7} \\
0 & 0 & 0 & C_{43}^{(5)} \Theta_{1} \Theta_{5} & 0
\end{array}\right)
\end{aligned}
$$

where

$$
C_{10}^{(5)}=C_{34}^{(5)}=\frac{H(2 \eta) \Theta(4 \eta)}{\Theta(2 \eta)}
$$




$$
\begin{aligned}
& C_{21}^{(5)}=C_{23}^{(5)}=\frac{H(2 \eta) \Theta(2 \eta)}{\Theta(0)} \\
& C_{32}^{(5)}=C_{12}=\frac{H(6 \eta) H(4 \eta) \Theta(6 \eta)}{H(2 \eta) \Theta(2 \eta)} \\
& C_{43}^{(5)}=C_{01}^{(5)}=\frac{H(8 \eta) \Theta^{2}(2 \eta)}{\Theta^{2}(4 \eta)}
\end{aligned}
$$

\section{The matrix $R^{(3)}(\mu, \nu)$}

For comparison we write $R^{(3)}(\mu, \nu)$ in the notation used above as

$$
R^{(3)}(v)(+,+)=\left(\begin{array}{ccc}
C_{00}^{(3)} \Theta_{0} H_{2} & 0 & C_{02}^{(3)} \Theta_{2} H_{0} \\
0 & C_{11}^{(3)} \Theta_{1} H_{1} & 0 \\
C_{20}^{(3)} \Theta_{0} H_{2} & 0 & C_{22}^{(3)} \Theta_{2} H_{0}
\end{array}\right)
$$

where

$$
\begin{aligned}
C_{00}^{(3)} & =C_{22}^{(3)}=\Theta^{2}(2 \eta) / \Theta(0) \\
C_{20}^{(3)} & =C_{02}^{(3)}=H^{2}(2 \eta) / \Theta(0) \\
C_{11}^{(3)} & =\Theta(4 \eta) \\
R^{(3)}(v)(+,-) & =\left(\begin{array}{ccc}
0 & C_{01}^{(3)} H_{1}^{2} & 0 \\
C_{10}^{(3)} \Theta_{0} \Theta_{2} & 0 & C_{12}^{(3)} H_{0} H_{2} \\
0 & C_{21}^{(3)} \Theta_{1}^{2} & 0
\end{array}\right),
\end{aligned}
$$

where

$$
\begin{aligned}
& C_{10}^{(3)}=C_{12}^{(3)}=H(2 \eta) \Theta(2 \eta) / \Theta(0) \\
& C_{01}^{(3)}=C_{21}^{(3)}=H(4 \eta)
\end{aligned}
$$

\section{Comments}

In the matrix $R^{(3)}(\mu, \nu)$ of (5.43), the coefficients $C_{01}^{(3)}=C_{21}^{(3)}$ vanish for $\eta=K / 2$; in the matrix $R^{(4)}(\mu, \nu)$ of (5.8), (5.13), the coefficients $C_{02}^{(4)}=C_{31}^{(4)}$ and $C_{01}^{(4)}=C_{32}^{(4)}$ vanish for $\eta=m_{1} K / 3$; and in the matrix $R^{(5)}(\mu, \nu)$ of (5.26), (5.34), the coefficients $C_{01}^{(5)}=C_{43}^{(5)}$ and $C_{02}^{(5)}=C_{42}^{(5)}$ vanish for $\eta=m_{1} K / 4$. Therefore the decomposition property (5.2) holds and it is now a simple matter to see that the functional equation (3.15) holds. This, of course, was the criteria used to obtain the similarity transformation matrices in the first place. 
What is not automatically guaranteed by our construction is that the transformed matrices $\tilde{R}^{(j)}(\mu, \nu)$ have matrix elements which depend only on one of the two linearly independent products of theta functions and that with the exception of $C_{22}^{(5)}$ all $C_{j k}^{(j)}$ are factored products of the theta functions $H(a \eta)$ and $\Theta(a \eta)$. These two properties make $\tilde{R}^{(j)}(\mu, \nu)$ much simpler for $j=4,5$ than the original matrices $R^{(j)}(\mu, \nu)$. It is our belief that these $\tilde{R}^{(j)}(\mu, \nu)$ are the "simplest possible" similarity transformations of $R^{(j)}(\mu, \nu)$ and that this is the form which should be generalized to arbitrary $j$.

\section{§6. Comparison with Sklyanin}

An alternative approach to the fusion matrices has been given by Sklyanin [13,14] who, instead of $R^{(j)}(\mu, \nu)$ considers the four matrices independent of the spectral variable $v S_{k}^{(j)}$ with $0 \leq k \leq 3$ which are defined by

$$
\begin{aligned}
& (a(v+(j-2) \eta))+b(v+(j-2) \eta)) S_{0}^{(j)} \\
& =R^{(j)}(+,+)(v)+R^{(j)}(-,-)(v) \\
& (a(v+(j-2) \eta))-b(v+(j-2) \eta)) S_{3}^{(j)} \\
& =R^{(j)}(+,+)(v)-R^{(j)}(-,-)(v) \\
& (c(v+(j-2) \eta))+d(v+(j-2) \eta)) S_{1}^{(j)} \\
& =R^{(j)}(+,-)(v)+R^{(j)}(-,+)(v) \\
& (c(v+(j-2) \eta))-d(v+(j-2) \eta)) S_{2}^{(j)} \\
& =R^{(j)}(+,-)(v)-R^{(j)}(-,+)(v)
\end{aligned}
$$

where $a(v), b(v), c(v)$ and $d(v)$ are defined by $(2.2)$.

Sklyanin shows that in order for $R^{(j)}(\mu, \nu)(v)$ to satisfy the Yang Baxter equation that the $S_{k}^{(j)}$ must be a representation of the algebra

$$
\begin{aligned}
& S_{\alpha} S_{0}-S_{0} S_{\alpha}=J_{\beta, \gamma}\left(S_{\beta} S_{\gamma}+S_{\gamma} S_{\beta}\right) \\
& S_{\alpha} S_{\beta}-S_{\beta} S_{\alpha}=\left(S_{0} S_{\gamma}+S_{\gamma} S_{0}\right)
\end{aligned}
$$

where $\alpha, \beta, \gamma$ are any cyclic permutation of $1,2,3$ and the structure constants $J_{\alpha, \beta}$ satisfy

$$
J_{12}+J_{23}+J_{31}+J_{12} J_{23} J_{31}=0
$$

and are explicitly computed in terms of theta functions. Sklyanin demonstrated that the finite dimensional representations of this algebra may be constructed from the space of theta functions with zero characteristics of order $j$ as defined in $[23]$. 
We have verified that the matrices $S_{k}^{(j)}$ constructed from our matrices $\tilde{R}^{(j)}(\mu, \nu)(v)$ do in fact satisfy Sklyanin's algebra although we are not aware of any direct proof that the fusion construction and the algebra (6.5) are equivalent. The relation of the functional equation (3.15) to Sklyanin's algebra also remains to be studied.

\section{$\S 7$. The Functional Equation for $Q$ at $L=2$}

We conclude by proving relation (3.10) for $L=2$.

$$
T^{(2)}(v-K)=e^{-i N \pi v / 2 K} Q_{R}(v) M^{-1} Q_{L}\left(v-i K^{\prime}\right) S .
$$

We proceed in two steps by first explicitly computing the matrix $M$ and then proving that (7.1) holds.

\section{A. Computation of $M$}

The conjecture for the functional equation for $Q$ in (3.1) is not complete because we have not given an explicit form for the normalizing matrix $A$. Therefore because $M$ is computed from $A$ by (3.8) our first task is to find a form for $M$ which is consistent with the conjecture. For $L=2$ we do this by setting $v=3 K / 2$ in $(3.9)$ to find

$$
M=\frac{\exp \left(-\frac{i 3 \pi N}{4}\right)}{h^{N}(K)} Q_{L}\left(3 K / 2-i K^{\prime}\right) Q_{R}(K / 2)
$$

The matrices $Q_{R}$ and $Q_{L}$ are defined by (2.3) and (2.7) where the matrices $S_{R}$ and $S_{L}$ contain the arbitrary parameters $\tau_{\alpha, m}$ which are restricted only by the requirement that the resulting matrices be nonsingular. We find it convenient to make the choice

$$
\tau_{\gamma, 0}=\delta_{\gamma,-1}, \quad \tau_{\gamma, 1}=\delta_{\gamma,-1}, \quad \tau_{\gamma,-1}=\delta_{\gamma, 1}, \quad \tau_{\gamma, 2}=\delta_{\gamma, 1}
$$

and thus we have explicitly from (2.4) and (2.8) with $L=2$

$$
\begin{aligned}
& S_{R}(+, \beta)(v)=\left(\begin{array}{ll}
H(v+K) \delta_{\beta,-1} & H(v) \delta_{\beta, 1} \\
-H(v) \delta_{\beta,-1} & -H(v+K) \delta_{\beta, 1}
\end{array}\right), \\
& S_{R}(-, \beta)(v)=\left(\begin{array}{ll}
\Theta(v+K) \delta_{\beta,-1} & \Theta(v) \delta_{\beta, 1} \\
\Theta(v) \delta_{\beta,-1} & \Theta(v+K) \delta_{\beta, 1}
\end{array}\right),
\end{aligned}
$$




$$
\begin{aligned}
& S_{L}(\alpha,+)(v)=\left(\begin{array}{ll}
H(v-K) \delta_{\alpha,-1} H(v) \delta_{\alpha, 1} \\
-H(v) \delta_{\alpha,-1} & -H(v-K) \delta_{\alpha, 1}
\end{array}\right), \\
& S_{L}(\alpha,-)(v)=\left(\begin{array}{ll}
\Theta(v-K) \delta_{\alpha,-1} & \Theta(v) \delta_{\alpha, 1} \\
\Theta(v) \delta_{\alpha,-1} & \Theta(v-K) \delta_{\alpha, 1}
\end{array}\right)
\end{aligned}
$$

Thus we find

$$
M_{\alpha \beta}=\frac{\exp \left(-\frac{i 3 \pi N}{4}\right)}{h^{N}(K)} \operatorname{Tr} m\left(\alpha_{1}, \beta_{1}\right) m\left(\alpha_{2}, \beta_{2}\right) \cdots m\left(\alpha_{N}, \beta_{N}\right)
$$

where

$$
\begin{aligned}
m(\alpha, \beta)= & S_{L}(\alpha,+)\left(3 K / 2-i K^{\prime}\right) S_{R}(+, \beta)(K / 2) \\
& +S_{L}(\alpha,-)\left(3 K / 2-i K^{\prime}\right) S_{R}(-, \beta)(K / 2) \\
= & -2 i q^{-1 / 4} \exp \left(\frac{i \pi}{4}\right) H(K / 2) \Theta(K / 2) \hat{m}(\alpha, \beta)
\end{aligned}
$$

with

$$
\hat{m}(\alpha, \beta)=\left(\begin{array}{llll}
\delta_{\alpha,-1} \delta_{\beta,-1} & \delta_{\alpha,-1} \delta_{\beta, 1} & i \delta_{\alpha, 1} \delta_{\beta,-1} & i \delta_{\alpha, 1} \delta_{\beta, 1} \\
0 & 0 & 0 & 0 \\
0 & 0 & 0 & 0 \\
i \delta_{\alpha,-1} \delta_{\beta,-1} & i \delta_{\alpha,-1} \delta_{\beta, 1} \delta_{\alpha, 1} \delta_{\beta,-1} & \delta_{\alpha, 1} \delta_{\beta, 1}
\end{array}\right)
$$

Only the first and the last column of $\hat{m}$ contribute to trace in (7.6) and thus

$$
M_{\alpha, \beta}=\left(-2 \frac{H(K / 2) \Theta(K / 2)}{q^{1 / 4} h(K)}\right)^{N} \operatorname{Tr}\left(\rho\left(\alpha_{1}, \beta_{1}\right) \cdots \rho\left(\alpha_{N}, \beta_{N}\right)\right)
$$

where

$$
\rho_{\alpha \beta}=\left(\begin{array}{ll}
\delta_{\alpha,-1} \delta_{\beta,-1} & i \delta_{\alpha, 1} \delta_{\beta, 1} \\
i \delta_{\alpha,-1} \delta_{\beta,-1} \delta_{\alpha, 1} \delta_{\beta, 1}
\end{array}\right)
$$

and

$$
M_{\alpha, \beta}^{-1}=\left(-2 \frac{H(K / 2) \Theta(K / 2)}{q^{1 / 4} h(K)}\right)^{-N} \operatorname{Tr}\left(\rho\left(\alpha_{1}, \beta_{1}\right) \cdots \rho\left(\alpha_{N}, \beta_{N}\right)\right)
$$

B. Computation of $e^{-i N \pi v / 2 K} Q_{R}(v) M^{-1} Q_{L}\left(v-i K^{\prime}\right) S$

We now use (2.19), (7.5), (7.6) and (7.11) in the right hand side of (7.1) to find

$$
\begin{aligned}
& \left.e^{-i N \pi v / 2 K} Q_{R}(v) M^{-1} Q_{L}\left(v-i K^{\prime}\right) S\right|_{\alpha, \beta} \\
& =\left(-\frac{h(K)}{2 H(K / 2) \Theta(K / 2)}\right)^{N} \operatorname{Tr} X\left(\alpha_{1}, \beta_{1}\right) \cdots X\left(\alpha_{N}, \beta_{N}\right)
\end{aligned}
$$


where

$$
\begin{aligned}
X_{\alpha, \beta}= & e^{-i \pi v / 2 K} q^{1 / 4} \\
& \times \sum_{\gamma, \lambda} S_{R}(\alpha, \gamma)(v) \rho(\gamma, \lambda) S_{L}(\lambda, \beta)\left(v+2 K-i K^{\prime}\right)
\end{aligned}
$$

which, using the notation,

$$
H_{K}=H(v+K), \quad \Theta_{K}=\Theta(v+K), \quad H=H(v), \quad \Theta=\Theta(v)
$$

is explicitly written as

$$
\begin{aligned}
& X(+,+)=\sum_{\gamma, \lambda}\left(\begin{array}{l}
H_{K} \delta_{\gamma,-1} H \delta_{\gamma, 1} \\
-H \delta_{\gamma,-1}-H_{K} \delta_{\gamma, 1}
\end{array}\right) \otimes\left(\begin{array}{ll}
\delta_{\gamma,-1} \delta_{\lambda,-1} & i \delta_{\gamma, 1} \delta_{\lambda, 1} \\
i \delta_{\gamma,-1} \delta_{\lambda,-1} & \delta_{\gamma, 1} \delta_{\lambda, 1}
\end{array}\right) \\
& \otimes\left(\begin{array}{ll}
\Theta_{K} \delta_{\lambda,-1} & i \Theta \delta_{\lambda, 1} \\
-i \Theta \delta_{\gamma,-1} & -\Theta_{K} \delta_{\gamma, 1}
\end{array}\right) \\
& \text { (7.16) } \quad X(-,-)=\sum_{\gamma, \lambda}\left(\begin{array}{cc}
\Theta_{K} \delta_{\gamma,-1} & \Theta \delta_{\gamma, 1} \\
\Theta \delta_{\gamma,-1} & \Theta_{K} \delta_{\gamma, 1}
\end{array}\right) \otimes\left(\begin{array}{cc}
\delta_{\gamma,-1} \delta_{\lambda,-1} & i \delta_{\gamma, 1} \delta_{\lambda, 1} \\
i \delta_{\gamma,-1} \delta_{\lambda,-1} \delta_{\gamma, 1} \delta_{\lambda, 1}
\end{array}\right) \\
& \otimes\left(\begin{array}{ll}
H_{K} \delta_{\lambda,-1} & -i H \delta_{\lambda, 1} \\
-i H \delta_{\gamma,-1} & H_{K} \delta_{\gamma, 1}
\end{array}\right) \\
& X(+,-)=\sum_{\gamma, \lambda}\left(\begin{array}{l}
H_{K} \delta_{\gamma,-1} H \delta_{\gamma, 1} \\
-H \delta_{\gamma,-1}-H_{K} \delta_{\gamma, 1}
\end{array}\right) \otimes\left(\begin{array}{ll}
\delta_{\gamma,-1} \delta_{\lambda,-1} & i \delta_{\gamma, 1} \delta_{\lambda, 1} \\
i \delta_{\gamma,-1} \delta_{\lambda,-1} & \delta_{\gamma, 1} \delta_{\lambda, 1}
\end{array}\right) \\
& \otimes\left(\begin{array}{ll}
H_{K} \delta_{\lambda,-1} & -i H \delta_{\lambda, 1} \\
-i H \delta_{\gamma,-1} & H_{K} \delta_{\gamma, 1}
\end{array}\right) \\
& \text { (7.18) } \quad X(-,+)=\sum_{\gamma, \lambda}\left(\begin{array}{cc}
\Theta_{K} \delta_{\gamma,-1} & \Theta \delta_{\gamma, 1} \\
\Theta \delta_{\gamma,-1} & \Theta_{K} \delta_{\gamma, 1}
\end{array}\right) \otimes\left(\begin{array}{cc}
\delta_{\gamma,-1} \delta_{\lambda-1} & i \delta_{\gamma, 1} \delta_{\lambda 1} \\
i \delta_{\gamma,-1} \delta_{\lambda,-1} \delta_{\gamma, 1} \delta_{\lambda, 1}
\end{array}\right) \\
& \otimes\left(\begin{array}{ll}
\Theta_{K} \delta_{\lambda,-1} & i \Theta \delta_{\lambda, 1} \\
-i \Theta \delta_{\gamma,-1}-\Theta_{K} \delta_{\gamma, 1}
\end{array}\right)
\end{aligned}
$$


We note that

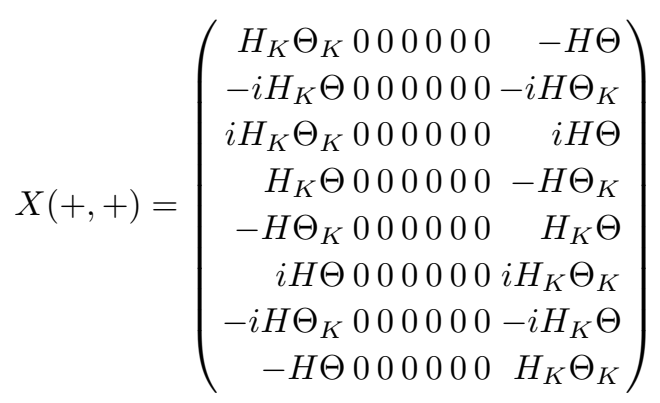

The rank of $X(+,+)$ is two and thus when used in the trace in (7.12) it may be replaced by

$$
Z(+,+)=\left(\begin{array}{cc}
H_{K} \Theta_{K} & -H \Theta \\
-H \Theta & H_{K} \Theta_{K}
\end{array}\right)
$$

Similarly $X(+,-), X(-,+)$, and $X(-,-)$ may be replaced by

$$
\begin{gathered}
Z(+,-)=\left(\begin{array}{rr}
H_{K}^{2} & H^{2} \\
-H^{2} & -H_{K}^{2}
\end{array}\right) \\
Z(-,+)=\left(\begin{array}{cc}
\Theta_{K}^{2} & -\Theta^{2} \\
\Theta^{2} & -\Theta_{K}^{2}
\end{array}\right) \\
Z(-,-)=\left(\begin{array}{cc}
\Theta_{K} H_{K} & \Theta H \\
\Theta H & \Theta_{K} H_{K}
\end{array}\right)
\end{gathered}
$$

and therefore we have

$$
\begin{aligned}
& \left.e^{-i N \pi v / 2 K} Q_{R}(v) M^{-1} Q_{L}\left(v-i K^{\prime}\right) S\right|_{\alpha, \beta} \\
& \quad=\left(-\frac{h(K)}{2 H(K / 2) \Theta(K / 2)}\right)^{N} \operatorname{Tr} Z\left(\alpha_{1}, \beta_{1}\right) \cdots Z\left(\alpha_{N}, \beta_{N}\right)
\end{aligned}
$$

\section{Proof of (7.1)}

We recall from (4.1) and (4.2) that

$$
\left.T^{(2)}\right|_{\alpha, \beta}(v-K)=\operatorname{Tr} R^{(2)}\left(\alpha_{1}, \beta_{1}\right)(v-K) \cdots R^{(2)}\left(\alpha_{N}, \beta_{N}\right)(v-K)
$$

with 


$$
\begin{aligned}
R^{(2)}(+,+)(v-K) & =\left(\begin{array}{cc}
a(v-K) & 0 \\
0 & b(v-K)
\end{array}\right), \\
R^{(2)}(+,-)(v-K) & =\left(\begin{array}{cc}
0 & d(v-K) \\
c(v-K) & 0
\end{array}\right), \\
R^{(2)}(-,+)(v-K) & =\left(\begin{array}{cc}
0 & c(v-K) \\
d(v-K) & 0
\end{array}\right), \\
R^{(2)}(-,-)(v-K) & =\left(\begin{array}{cc}
b(v-K) & 0 \\
0 & a(v-K)
\end{array}\right)
\end{aligned}
$$

We thus complete the proof of (7.1) by noting that there is a similarity transformation by a matrix $G$ which maps the matrices $Z(\alpha, \beta)$ on the matrices $R^{(2)}(\alpha, \beta)$. Specifically

$$
\begin{aligned}
G Z(+,+) G^{-1} & =-\frac{H(K)}{H(K / 2) \Theta(K / 2)}\left(\begin{array}{cc}
a(v-K) & 0 \\
0 & b(v-K)
\end{array}\right) \\
G Z(+,-) G^{-1} & =-\frac{H(K)}{H(K / 2) \Theta(K / 2)}\left(\begin{array}{cc}
0 & d(v-K) \\
c(v-k) & 0
\end{array}\right) \\
G Z(-,+) G^{-1} & =-\frac{H(K)}{H(K / 2) \Theta(K / 2)}\left(\begin{array}{cc}
0 & c(v-K) \\
d(v-k) & 0
\end{array}\right) \\
G Z(-,-) G^{-1} & =-\frac{H(K)}{H(K / 2) \Theta(K / 2)}\left(\begin{array}{cc}
b(v-K) & 0 \\
0 & a(v-K)
\end{array}\right)
\end{aligned}
$$

where

$$
G=\left(\begin{array}{rr}
a_{11} & a_{11} \\
-a_{22} & a_{22}
\end{array}\right) \quad G^{-1}=\left(\begin{array}{rr}
a_{22} & -a_{11} \\
a_{22} & a_{11}
\end{array}\right)
$$

with

$$
a_{11}=\frac{1}{\sqrt{2}}\left(\frac{H(K / 2)}{\Theta(K / 2)}\right)^{1 / 2} \quad a_{22}=\frac{1}{\sqrt{2}}\left(\frac{\Theta(K / 2)}{H(K / 2)}\right)^{1 / 2}
$$

The verification of this similarity transformation is straightforward by use of identities such as

$$
\begin{aligned}
H(v) \Theta(v)+H(v+K) \Theta(v+K) & =\frac{H(K) \Theta(K)}{H(K / 2) \Theta(K / 2)} H(v+K / 2) \Theta(v-K / 2) \\
& =-\frac{H(K)}{H(K / 2) \Theta(K / 2)} b(v-K),
\end{aligned}
$$




$$
\begin{aligned}
H(v+K) \Theta(v+K)-H(v) \Theta(v)= & -\frac{H(K) \Theta(K)}{H(K / 2) \Theta(K / 2)} H(v-K / 2) \Theta(v+K / 2) \\
& -\frac{H(K)}{H(K / 2) \Theta(K / 2)} a(v-K)
\end{aligned}
$$

and

$$
\begin{aligned}
H^{2}(v+K)+H^{2}(v) & =\frac{H^{2}(K)}{\Theta^{2}(K / 2)} \Theta(v+K / 2) \Theta(v-K / 2) \\
& =\frac{H(K)}{\Theta^{2}(K / 2)} c(v-K) .
\end{aligned}
$$

Thus using (7.27)-(7.30) and (7.25) in (7.24) and recalling the definition (2.13) of $h(v)$ we find

$\left.e^{-i N \pi v / 2 K} Q_{R}(v) M^{-1} Q_{L}\left(v-i K^{\prime}\right) S\right|_{\alpha, \beta}=\left(\frac{\Theta(0) H^{2}(K) \Theta(K)}{2 H^{2}(K / 2) \Theta^{2}(K / 2)}\right)^{N} T_{\alpha, \beta}^{(2)}(v-K)$

Thus if we finally use the identity

$$
\frac{\Theta(0) H^{2}(K) \Theta(K)}{2 H^{2}(K / 2) \Theta^{2}(K / 2)}=1
$$

which follows from (4.5) with $a=K, v=K / 2$ and $u=-K / 2$ we see that (7.1) is proven.

\section{Appendix A. Theta Functions}

The definition of Jacobi Theta functions of nome $q$ is

$$
\begin{aligned}
H(v) & =2 \sum_{n=1}^{\infty}(-1)^{n-1} q^{\left(n-\frac{1}{2}\right)^{2}} \sin [(2 n-1) \pi v /(2 K)] \\
\Theta(v) & =1+2 \sum_{n=1}^{\infty}(-1)^{n} q^{n^{2}} \cos (n v \pi / K) \\
& =-i q^{1 / 4} e^{\pi i v /(2 K)} H\left(v+i K^{\prime}\right)
\end{aligned}
$$

where $K$ and $K^{\prime}$ are the standard elliptic integrals of the first kind and

$$
q=e^{-\pi K^{\prime} / K}
$$


These theta functions satisfy

$$
\Theta(v)=\Theta(-v), \quad H(v)=-H(-v)
$$

and the quasi periodicity relations (15.2.3) of ref. [24]

$$
\begin{aligned}
H(v+2 K) & =-H(v) \\
H\left(v+2 i K^{\prime}\right) & =-q^{-1} e^{-\pi i v / K} H(v)
\end{aligned}
$$

and

$$
\begin{aligned}
\Theta(v+2 K) & =\Theta(v) \\
\Theta\left(v+2 i K^{\prime}\right) & =-q^{-1} e^{-\pi i v / K} \Theta(v) .
\end{aligned}
$$

From (A.2) we see that $\Theta(v)$ and $H(v)$ are not independent but satisfy (15.2.4) of ref. [24].

$$
\begin{gathered}
\Theta\left(v \pm i K^{\prime}\right)= \pm i q^{-1 / 4} e^{\mp \frac{\pi i v}{2 K}} H(v) \\
H\left(v \pm i K^{\prime}\right)= \pm i q^{-1 / 4} e^{\mp \frac{\pi i v}{2 K}} \Theta(v) .
\end{gathered}
$$

\section{Acknowledgements}

We are pleased to thank Prof. T. Deguchi, Prof. M. Jimbo, Prof. T. Miwa, Prof. R. I. Nepomechie, and Prof. N. Yu. Reshetikhin for fruitful discussions. We are particularly indebted to Prof. R. J. Baxter for information on theta functions and for bringing the functional equation (3.15) to our attention. This work is partially supported by NSF grant DMR0302758.

\section{References}

[1] Baxter, R. J., Partition function of the eight vertex lattice model, Ann. Phys., 70 (1972), 193-228.

[2] Au-Yang, H., McCoy, B. M., Perk, J. H. H., Tang, S. and Yan, M-L., Commuting transfer matrices in the chiral Potts model: solutions of the star triangle equations with genus $>1$, Phys. Lett. A, 123 (1987), 219-223.

[3] Bazhanov, V. V. and Stroganov, Yu. G., Chiral Potts model as a descendant of the six-vertex model, J. Stat. Phys., 59 (1990), 799-817.

[4] Albertini, G., McCoy, B. M. and Perk, J. H. H., Commensurate-incommensurate transition in the ground state of the superintegrable chiral Potts model, Phys. Lett. A, 135 (1989), 159-166.

[5] Eigenvalue spectrum of the superintegrable chiral Potts model, Adv. Stud. Pure Math., 19, ed. Jimbo, M., Miwa, T. and Tsuchiya, A., Kinikuniya-Academic Press, Tokyo, (1989), 1-55. 
[6] Baxter, R. J., Bazhanov, V. V. and Perk, J. H. H., Functional relations for transfer matrices of the chiral Potts model, Int. J. Mod. Phys. B, 4 (1990), 803-870.

[7] Baxter, R. J., Eight-vertex model in lattice statistics and one-dimensional anisotropic Heisenberg chain I: Some fundamental eigenvectors, Ann. Phys., 76 (1973), 1-24.

[8] _ Eight-vertex model in lattice statistics and one-dimensional anisotropic Heisenberg chain II: Equivalence to a generalized Ice-type lattice model, Ann. Phys., 76 (1973), $25-47$.

[9] _ Eight-vertex model in lattice statistics and one--dimensional anisotropic Heisenberg chain III: Eigenvectors of the transfer matrix and the Hamiltonian, Ann. Phys., 76 (1973), 48-71.

[10] Fabricius, K. and McCoy, B. M., New developments in the eight vertex model, J. Stat. Phys., 111 (2003), 323-337.

[11] Kulish, P. P. and Reshetikhin, N. Yu., Quantum linear problem for the sine-Gordon and higher representations, J. Soviet Math., 23 (1983), 2435; translated from Zap. Nauch. Sem., LOMI, 101 (1981), 101-110.

[12] Kulish, P. P., Reshetikhin, N. Yu. and Sklyanin, E. K., Yang Baxter equation and representation theory, Lett. Math. Phys., 5 (1981), 393-403.

[13] Sklyanin, E. K., Some algebraic structures connected with the Yang-Baxter equation, Funct. Anal. Appl., 16 (1982), 27-34.

[14] Some algebraic structures connected with the Yang-Baxter equation. Representations of quantum algebras, Funct. Anal. Appl., 17 (1984), 34-48.

[15] Date, E., Jimbo, M., Miwa, T. and Okado, M., Fusion of the eightvertex SOS model, Lett. Math. Phys., 12 (1986), 209-215.

[16] Kirillov, A. N. and Reshetikhin N. Yu., Exact solution of the integrable XXZ Heisenberg model with arbitrary spin I. The ground state and the excitation spectrum, J. Phys. A, 20 (1987), 1565-1585.

[17] Date, E., Jimbo, M., Kuniba, A., Miwa, T. and Okado, M., Exactly solvable SOS models, II. Proof of the star triangle relation and combinatorial identities, Adv. Stud. Pure Math., 16 (1988), 17-122.

[18] Nepomechie, R. I., Solving the open $X X Z$ spin chain with nondiagonal boundary terms at roots of unity, Nucl. Phys. B, 622 (2002), 615-632.

[19] , Functional relations and Bethe ansatz for the $X X Z$ chain, J. Stat. Phys., 111 (2003), 1363-1376.

[20] , Bethe ansatz solution of the open $X X Z$ chain with nondiagonal boundary terms, hepth/0304092.

[21] Bazhanov, V. V. and Reshetikhin, N. Yu., Critical RSOS models and conformal field theory, Int. J. Mod. Phys. A, 4 (1989), 115-142.

[22] Baxter, R. J., private communication.

[23] Weber, H., Lehrbuch der Algebra, B.III. Elliptische Funktionen und Algebraische Zahlen, F. Vieweg und Sohn, Braunschweig, 1908.

[24] Baxter, R. J., Exactly solved models, Academic Press, London, 1982. 